\title{
Tecnologia a favor da Educação: Um Estudo de Caso das Escolas do Espírito Santo
}

\author{
Marcelo Otone Aguiar ${ }^{1}$ e Edilson Luiz do Nascimento ${ }^{2}$ \\ ${ }^{1}$ Departamento de Sistemas de Informação \\ UCL - Associação de Ensino Superior Unificado do Centro Leste, ES - Brasil \\ ${ }^{2}$ Gerência de Ensino Uned Serra, Coordenadoria de Informática \\ Instituto Federal de Educação, Ciência e Tecnologia do Espírito Santo - Brasil \\ marcelootoneducl.br, edilson@ifes.edu.br
}

\begin{abstract}
Previous studies indicate that the use the use of technology in education favors the autonomy of teachers and students and provides an educational environment in which the student ceases to be passive and starts to act critically. This study examined the relationship between the presence of information and communication technology in high schools of the Espirito Santo with its performance, measured by ENEM. Found results in a positive relationship by about $41 \%$, ie, the schools attended by the information and communication technology were superior performance to other schools in the Espírito Santo.
\end{abstract}

Resumo. Estudos anteriores apontam que o uso da tecnologia na educação favorece a autonomia dos professores e alunos e propicia um ambiente educacional em que o aluno deixa de ser passivo e passa a atuar de forma crítica. Este estudo analisou a relação entre a presença de tecnologia de informação e comunicação em escolas de ensino médio do Espírito Santo com o seu desempenho, medido através do ENEM. Obteve-se como resultado a existência de relação positiva em cerca de 41\%, ou seja, as escolas com a presença de tecnologia da informação e comunicação apresentaram desempenho superior às demais escolas no Espírito Santo.

\section{Introdução}

Ao passo que as Tecnologias da Comunicação e Informação têm evoluído, a educação está absorvendo boa parte dos recursos tecnológicos que chegam ao mercado, inclusive muitos destes recursos tecnológicos, são desenvolvidos especialmente para a área da educação, como é o caso das lousas digitais, já presentes em muitas escolas (CALDAS; NOBRE; GAVA, 2011). Este movimento está ocorrendo porque os agentes da educação estão percebendo a importância do uso de tecnologia no ambiente escolar. Uma vez que vivemos em uma sociedade da informação, é importante que a educação acompanhe esta nova era altamente dependente da tecnologia. (LOPES et al. 2010; LÖBLER et al. 2012).

Várias são as motivações que levam os agentes, responsáveis pela educação, a recorrer à TIC como ferramenta aliada a esta nova educação que está surgindo. Corrêa et al. (2006), em um estudo realizado para avaliar a implantação de computadores portáteis em ambiente escolar, apontaram como resultado de sua pesquisa, a ocorrência de uma boa aceitabilidade da proposta do projeto pelos alunos e os professores, além disso, foi possível observar um aumento na variedade de experiências educacionais. 
Outra pesquisa realizada para o mesmo projeto apontou que os alunos ficaram mais atentos às aulas convencionais e que houve um aumento no retorno das atividades propostas pelos professores (FRANCO et al. 2007).

Löbler et al. (2012) avaliaram a relação entre a presença de laboratórios de informática com o desempenho das escolas. Para isso, os autores escolheram de forma não aleatória, duas escolas sendo uma com alto desempenho e outra com baixo desempenho, aplicaram questionários visando identificar pontos determinantes em relação ao uso do laboratório de informática nessas escolas. Os autores não encontraram relação entre o desempenho dos alunos com o uso do computador, mas observaram que o simples uso dos computadores não é suficiente para aumentar o desempenho.

Valente (1997) em seu artigo sobre "O uso inteligente do computador na educação" salienta que utilizar a tecnologia da informação de forma inteligente na educação pode promover a autonomia dos professores e alunos e flexibilizar o atual sistema tradicional da educação. O autor diz ainda que tirar o proveito inteligente da tecnologia favorecerá um ambiente educacional em que o aluno deixa de ser passivo e passa a atuar de forma crítica e capaz de sobreviver na atual sociedade. As palavras de Valente nos remetem a duas perguntas: Os investimentos realizados em tecnologia para a educação têm contribuído para o avanço da educação? É possível que já estejamos colhendo frutos dos esforços que tem sido feitos com o apoio da tecnologia da informação?

Para responder a estas perguntas, este estudo analisou quantitativamente, por meio de inferências estatísticas se a implantação de recursos tecnológicos, em escolas de ensino médio no Espirito Santo, está contribuindo para o desenvolvimento da educação. Para essa análise, foram coletados dados do Censo das escolas de ensino médio e os dados do ENEM (exame nacional do ensino médio). De acordo com Travitzki (2013) o ENEM sofreu mudanças consideráveis em seu formato a partir de 2009, sendo assim optou-se por realizar este pesquisa com os períodos de 2009, 2010 e 2011, naturalmente o mesmo período foi adotado para os dados do Censo.

A luz do que disse Valente (1997), Corrêa et al. (2006), FRANCO et al. (2007), LOPES et al. (2010) entre outros estudiosos da área de informática na educação, encontrou-se evidências estatísticas da existência de uma relação positiva entre as escolas de ensino médio do Espírito Santo em que foram implantados os recursos de tecnologia de informação e comunicação com o seu desempenho, medido através do ENEM. Além disso, esta pesquisa trouxe evidências de que as escolas públicas têm em média 141 pontos a menos que as escolas privadas com os mesmos níveis de computadores, disponibilidade de internet e laboratório de informática.

\section{Metodologia}

O objetivo desta pesquisa é obter evidências estatísticas da existência de uma relação positiva entre a disponibilidade de tecnologia digital nas escolas de ensino médio do Espírito Santo com o seu desempenho. Este estudo analisou quantitativamente se a implantação de recursos tecnológicos em escolas de ensino médio no Espírito Santo estão contribuindo para o desempenho dos alunos. Para que essa análise seja possível, foram coletados dados do Censo das escolas de ensino médio no Espírito Santo e os dados do ENEM, ambos referentes aos períodos de 2009, 2010 e 2011.

Para realizar esta análise de relação, foi adotado o método estatístico regressão 
linear. Conforme Levine et al. (2008) o método da regressão linear permite prever, com base em um modelo, valores de uma variável numérica em função do valor de outras variáveis. A regressão linear permite também uma análise do peso da relação entre as variáveis e quantificar os efeitos provenientes de mudanças nessas variáveis (LENINE, 2000; WOOLDRIDGE, 2006).

\subsection{Tabulação dos Dados Coletados}

Os dados do Censo foram disponibilizados pela INEP (2013) em formato texto, juntamente com o layout para leitura do arquivo. Inicialmente, foi realizada uma análise comparativa entre os layouts, referentes aos anos 2005, 2006, 2007, 2008, 2009, 2010 e 2011, com o intuito de verificar a compatibilidade entre a pesquisa do Censo Escolar realizada para estes anos. Os anos de 2005 e 2006 apresentaram diferenças substanciais com as pesquisas dos anos subsequentes. De forma que, as diferenças encontradas inviabilizaram o uso do Censo de 2005 e 2006.

O próximo passo foi analisar os dados do ENEM, neste caso, um importante estudo científico realizado com o ENEM, deu o suporte necessário para selecionar o período a ser avaliado. O estudo em questão foi realizado por Travitzki (2013) com o objetivo de analisar o ENEM como indicador de qualidade escolar. Dentre os resultados apresentados pelo autor, está à mudança estrutural que o ENEM sofreu a partir de 2009, inclusive mudanças relacionadas às habilidades e competências avaliadas pelo exame. Em função dessas mudanças optou-se por restringir o período avaliado a partir de 2009, sendo assim, foram incluídos no estudo os anos de 2009, 2010 e 2011.

\subsection{Modelo Estatístico}

Foram desenvolvidos 2 modelos matemáticos para este estudo. O primeiro modelo leva em consideração os principais fatores relacionados à presença de informática na escola, como laboratório de informática (LOPES et al., 2010 LÖBLER et al., 2012), computadores para os alunos (VALENTE, 1997; CORREAA et al., 2006; FRANCO et al., 2007) e internet (NERI, 2003; BIONDI; FELÍCIO, 2007). O segundo modelo leva em consideração os fatores do primeiro modelo e adicionalmente considera também se a escola é pública ou privada. Além disso, os 2 modelos tem como variável dependente o ENEM (GOMES; BORGES, 2009b; AZEVEDO, 2009; TRAVITZKI, 2013), que neste caso, é medido pela média aritmética das notas das provas objetivas realizadas pelos alunos para uma determinada escola conforme a equação 1 e a média aritmética entre as quatro médias obtidas referentes às provas de ciências da natureza, ciências humanas, linguagens e códigos e matemática conforme a equação 2 .

A equação 1 possibilitou calcular a média de cada uma das 4 provas para uma determinada escola em um ano. A equação 2, por sua vez, permitiu calcular a média final entre as 4 médias que foram calculadas para as 4 provas, tendo assim como resultado final uma média única para cada escola em um determinado ano. O resultado final deste cálculo deu origem à variável que foi denominada ENEM nesta pesquisa.

$$
\text { mediaNota }_{i t}=\frac{\sum_{i=0}^{n} \text { nota }_{i t}}{n}
$$

Em que:

mediaNota $_{i t}=\quad$ Resultado da média aritmética para uma observação $\boldsymbol{i}$ de um 

$n o t a_{i t}=\quad$ É a nota da prova realizada pelo aluno de uma determinada escola $\boldsymbol{i}$ no período $\boldsymbol{t}$
$n=$
É a quantidade de provas realizadas pelos alunos de uma determinada escola $\boldsymbol{i}$ no período $\boldsymbol{t}$.

$$
E N E M_{i t}=\frac{\sum_{i=0}^{n} \text { mediaNota }_{i t}}{n}
$$

Em que:

$E N E M_{i t}=\quad$ Resultado da média aritmética entre as médias calculadas para as quatro provas objetivas de uma escola $\boldsymbol{i}$ em um período $\boldsymbol{t}$;

mediaNota $_{i t}=\quad \hat{E}$ a nota média da escola em determinada prova objetiva $\boldsymbol{i}$ no período $\boldsymbol{t}$.

$n=$

As demais variáveis do primeiro modelo são de controle e responsáveis apenas por permitir que a análise estatística considere os diferentes anos dos dados que serão analisados (WOOLDRIDGE, 2006, p. 439), note que no modelo foram incluídos apenas os anos de 2009 e 2010, isso acontece porque são variáveis binárias, de forma que a ausência do ano 2009 e do ano 2010 indica a presença do ano 2011, não sendo assim necessária uma variável para este ano no modelo. A equação 3 representa o primeiro modelo matemático que foi utilizado na regressão linear.

$$
\begin{aligned}
& \text { ENEM }_{i t}=\beta_{i t}+\beta_{1} \text { Comp }_{i t}+\beta_{2} \text { internet }_{i t}+\beta_{3} \text { labInf }_{i t}+\beta_{4} \text { ano2009 }_{i t}+ \\
& \beta_{5}{\text { ano } 2010_{i t}}
\end{aligned}
$$

Em que:

$$
\begin{aligned}
& E N E M_{i t}=\quad \text { Dados do ENEM para uma respectiva escola } \boldsymbol{i} \text { em um período } \boldsymbol{t} \text {; } \\
& \beta_{i t}=\quad \text { É o intercepto da escola } \boldsymbol{i} \text { no período } \boldsymbol{t} \text {; } \\
& \beta_{1} n \text { Comp }_{i t}=\quad \text { É a quantidade de computadores para os alunos na escola } \boldsymbol{i} \text { no } \\
& \text { período } \boldsymbol{t} \text {. } \\
& \beta_{2} \text { internet }_{i t}=\quad \text { É a presença de internet banda larga de uma escola } \boldsymbol{i} \text { no período } \boldsymbol{t} \text {. } \\
& \beta_{3} \text { labInf } f_{i t}=\quad \text { Indica a presença de um laboratório de informática em uma escola } \\
& \boldsymbol{i} \text { no período } \boldsymbol{t} \text {. } \\
& \beta_{4} \text { ano2009 }_{i t}=\quad \text { Indica quando os dados da escola } \boldsymbol{i} \text { no período } \boldsymbol{t} \text { se referem a } \\
& 2009 . \\
& \beta_{5} \text { ano } 2010_{i t}=\quad \text { Indica quando os dados da escola } \boldsymbol{i} \text { no período } \boldsymbol{t} \text { se referem a } \\
& 2010 \text {. }
\end{aligned}
$$

O segundo modelo é similar ao primeiro, a diferença é que este modelo leva em consideração se a escola é pública ou não. O estudo de Azevedo (2009) trouxe evidências da importância de realizar este controle em um modelo estatístico visto que 
pode haver uma diferença substancial nos fatores envolvidos nas escolas privadas e públicas. A equação 4 representa o segundo modelo matemático que foi utilizado na regressão linear.

$$
\begin{aligned}
& \text { ENEM }_{i t}=\beta_{i t}+\beta_{1} \text { Comp }_{i t}+\beta_{2} \text { internet }_{i t}+\beta_{3} \text { labInf }_{i t}+\beta_{4} \text { ano2009 }_{i t}+ \\
& \beta_{5} \text { ano2010 }_{i t}+\beta_{6} \text { ESCPublica }_{i t}
\end{aligned}
$$

Em que:

$$
\begin{array}{ll}
E N E M_{i t}= & \begin{array}{l}
\text { Dados do ENEM para uma respectiva escola } \boldsymbol{i} \text { em um período } \boldsymbol{t} ; \\
\beta_{i t}=
\end{array} \\
\beta_{1} \text { EComp } & \begin{array}{l}
\text { É o intercepto da escola } \boldsymbol{i} \text { no período } \boldsymbol{t} ; \\
\text { É a quantidade de computadores para os alunos na escola } \boldsymbol{i} \text { no } \\
\text { período } \boldsymbol{t} .
\end{array} \\
\beta_{2} \text { internet }_{i t}= & \begin{array}{l}
\text { É a presença de internet banda larga de uma escola } \boldsymbol{i} \text { no período } \boldsymbol{t} . \\
\beta_{3} \text { labInf }_{i t}=
\end{array} \\
\begin{array}{l}
\text { Indica a presença de um laboratório de informática em uma escola } \\
\beta_{4} \text { ano2009 }_{i t}=
\end{array} & \begin{array}{l}
\text { Indica quando os dados da escola } \boldsymbol{i} \text { no período } \boldsymbol{t} \text { se referem a } \\
\text { 2009. }
\end{array} \\
\beta_{5} \text { ano2010 }_{i t}= & \text { Indica quando os dados da escola } \boldsymbol{i} \text { no período } \boldsymbol{t} \text { se referem a } \\
2010 . & \text { Indica se a escola } \boldsymbol{i} \text { no período } \boldsymbol{t} \text { é pública. } \\
\beta_{6} \text { ESCPublica }_{i t}= &
\end{array}
$$

Com o objetivo de avaliar se a implantação de recursos tecnológicos em escolas de ensino médio no Espírito Santo está contribuindo para o desenvolvimento da educação (VALENTE, 1997; NERI, 2003; CORRÊA et al. 2006; FRANCO et al. 2007) foi formulada a hipótese:

- $\mathrm{H}_{0}$ : A presença de recursos de tecnologia digital em escola é positivamente relacionada ao seu desempenho.

A impossibilidade de rejeitar essa hipótese indica que haverá evidências estatísticas de que quanto mais recursos de tecnologia de informação e comunicação houver em uma determinada escola, maior será o seu desempenho.

\section{Resultados}

Ao todo, foram extraídas das bases do Censo e do ENEM 1.417 observações das escolas do Espírito Santo para os anos de 2009, 2010 e 2011. Os dados incluem as variáveis necessárias aos modelos matemáticos que foram executados para a análise da relação entre a presença de tecnologia da informação e comunicação nas escolas com o seu desempenho. Como mencionado, a nota do ENEM para uma determinada escola foi calculada com base na média das notas obtidas pelos alunos da mesma. Conforme apresentado nas equações 3 e 4, essa variável foi utilizada como variável dependente.

Levine (2008) descreve os 4 pressupostos que devem ser mantidos para que possamos fazer conclusões acerca dos modelos regredidos com o método da regressão linear, sendo eles os pressupostos da linearidade, da independência, da normalidade e da homoscedasticidade. Levine (2008) salienta também a necessidade da análise da colinearidade em modelos de regressão linear múltipla. Desta forma, realizou-se para os dois modelos os testes necessários para análise destes pressupostos e da colinearidade. A 
conclusão geral sobre a análise dos pressupostos é que os dados se ajustam ao modelo e que as inferências estatísticas para os resultados são válidas. A seguir são apresentados os resultados do método da regressão linear para cada modelo matemático na Tabela 1 .

Tabela 1: Quadro geral dos resultados das regressões lineares

Os modelos foram regredidos com a correção robusta de White (WOOLDRIDGE, 2006). A variável dependente em cada regressão é o ENEM que foi definida com base na média das notas das provas objetivas do exame nacional do ensino médio (ENEM).

\begin{tabular}{lcc}
\hline Variável Independente & $\begin{array}{c}\text { Modelo 1 } \\
\text { (ENEM) }\end{array}$ & $\begin{array}{c}\text { Modelo 2 } \\
\text { (ENEM) }\end{array}$ \\
\hline nComp & $0,32956^{* * * *}$ & $0,35002^{* * * *}$ \\
& $(0,08302)$ & $(0,05307)$ \\
internet & $46,2210^{* * *}$ & 20,37678 \\
& $(18,2422)$ & $(16,0538)$ \\
labInf & $-26,3640^{*}$ & 4,76006 \\
& $(14,2523)$ & $(10,5086)$ \\
ano2009 & $-38,0193^{* * *}$ & $-40.2244 * * *$ \\
& $(6,83004)$ & $(5,3159)$ \\
ano2010 & $11,8265^{*}$ & $12,2267^{* *}$ \\
& $(6,67054)$ & $(5,35343)$ \\
EscPublica & - & $-141.809^{* * *}$ \\
\hline Tamanho da Amostra & 1.408 & $(4,85459)$ \\
Estatística F & 16,44 & 1.408 \\
Significância do Modelo & 0,00 & 178,42 \\
$\mathrm{R}^{2}$ & 0,0624 & 0,00 \\
$\mathrm{R}^{2}$ Ajustado & 0,0590 & 0,4173 \\
\hline Fonto O própio aus & & 0,4148 \\
\hline
\end{tabular}

Fonte: O próprio autor.

Nota: Erro padrão em parênteses * $p<0,10,{ }^{* *} p<0,05,{ }^{* * *} p<0,01$

\subsection{Análise da variável nComp}

Ao observar a primeira variável, nComp, podemos inferir algumas conclusões. A primeira é que o coeficiente " 0,32956 " e " 0,35002 " nos dois modelos é positivo, isso quer dizer que o número de computadores está relacionado positivamente com o desempenho, ou seja, um número maior de computadores está relacionado a um maior desempenho da escola. Essa é a relação esperada, pois confirma a hipótese deste estudo e a afirmação de autores como Valente (1997) Corrêa et al. (2006) e Franco et al. (2007) de que o uso de computadores possibilita melhoras no aprendizado dos alunos. Outra conclusão que podemos tirar a respeito do coeficiente é que ele é estatisticamente significante ao nível de $1 \%$ nos dois modelos, como pode ser visto na Tabela 1 . Por fim, analisando do ponto de vista econômico o estimador traz um resultado razoável, pois mantendo outros fatores fixos, se uma escola teve um ganho de 1 ponto em seu desempenho, o número de computadores desta escola aumentou em 0,33 e 0,35 em média no primeiro e segundo modelos, respectivamente.

\subsection{Análise da variável internet}

Similar à variável $n$ Comp, a variável internet, também apresentou uma relação positiva nos dois modelos, ou seja, uma maior disponibilidade de internet está relacionada ao maior desempenho dos alunos e consequentemente da escola. No entanto, a variável apresentou significância estatística apenas no primeiro modelo. Se mantiver outros 
fatores fixos, e a escola obter um ganho de 1 ponto em seu desempenho, a disponibilidade de internet aumentou em, por exemplo, 46,2210, no primeiro modelo, como essa unidade não está percentual, podemos concluir que em termos práticos essa informação não é muito útil, não sendo assim economicamente significante.

\subsection{Análise da variável $\operatorname{labInf}$}

A variável labInf, responsável por indicar a presença de um laboratório de informática, apresentou significância estatística ao nível de $10 \%$ no primeiro modelo, mas não houve significância estatística no segundo modelo. No primeiro modelo, a variável, apresentou uma relação negativa com a nota da escola medida pelo ENEM e no segundo modelo apresentou uma relação positiva, que é a relação esperada pela hipótese do estudo, mas como não há significância estatística da variável no segundo modelo, não se pode concluir nada, de forma que, não há evidência estatística de que o laboratório de informática contribua para o desempenho das escolas do Espírito Santo. Essa diferença da relação entre os modelos 1 e 2 é uma evidência da necessidade da inclusão da informação de escola pública ou privada em análises estatísticas com estudos realizados com escolas (AZEVEDO, 2009).

\subsection{Análise da variável EscPublica}

Pode-se afirmar que esta variável causou um grande impacto demonstrando assim sua necessidade. Por exemplo, ao analisarmos o campo $\mathrm{R}^{2}$, responsável por determinar o quão bem a reta de regressão se ajusta aos dados, notamos que o modelo 1 , sem a variável EscPublica, apresentou o valor 0,0624 ou 6,24\%. No modelo 2, em que houve a inclusão da variável EscPublica, o $\mathrm{R}^{2}$ calculado é $41,73 \%$, ou seja, a relação linear ficou muito superior com a inclusão da variável EscPublica.

Outra conclusão que podemos tirar é que esta variável apresentou uma relação negativa com a variável dependente, ENEM, o que significa que, pelo modelo 2, as escolas públicas tem em média 141,81 pontos, conforme o seu coeficiente, a menos que as escolas privadas com os mesmos níveis de computadores, disponibilidade de internet e laboratório de informática. Por fim, o estimador EscPublica se mostrou significante estatisticamente ao nível de $1 \%$ e economicamente significante, pois os valores apresentados por seu coeficiente se mostraram eficientes para conclusões acerca do desempenho entre escolas públicas e privadas.

\subsection{Resultados Gerais dos Modelos}

A Tabela 1 apresenta um resultado geral acerca do método da regressão linear aplicado aos dados para cada modelo estabelecido com o objetivo de validarmos a hipótese desta pesquisa. De uma forma geral, os 2 modelos apresentaram significância estatística, quando considerado o modelo como um todo. Isso pode ser observado na tabela 1 no campo significância do modelo, em que o valor " 0,00 ", resultado dos dois modelos, indica que a probabilidade de rejeitarmos a hipótese deste estudo é menor que $1 \%$.

Além da significância estatística, o segundo modelo apresentou um bom resultado de relação entre as variáveis dependente e independente. $\mathrm{O}$ primeiro modelo apresentou uma relação de 0,0624 como pode ser visto na Tabela 1 , no campo $\mathrm{R}^{2}$, ou seja, em média 6,24\% da variação no desempenho do exame do ENEM (desempenho das escolas) é explicado pelo modelo. O segundo modelo apresentou um $\mathrm{R}^{2}$ de 0,4173 , 
ou seja, em média 41,73\% da variação no desempenho do exame do ENEM é explicado por este modelo. Conclui-se que o percentual do segundo modelo é bom, visto que, os fatores que influenciam a educação vão muito além dos fatores observados neste estudo.

\section{Conclusão}

Este estudo buscou, por meio de inferências estatísticas, validar o que autores como Valente (1997), Neri (2003), Corrêa et al. (2006), Franco et al. (2007) e Löbler et al. (2012) apontaram sobre o uso de tecnologia em escolas do Espírito Santo. De uma forma geral, buscou-se evidências da existência ou não de uma relação entre a presença de tecnologia da informação e comunicação nas escolas com o seu desempenho. Para isso, utilizou a base de dados do Censo, pois nesta base estão presentes as informações de infraestrutura das escolas, inclusive infraestrutura relacionada à tecnologia. $\mathrm{O}$ desempenho das escolas foi extraído por meio das notas do ENEM, pois o objetivo foi medir o desempenho em termos de qualidade do ensino e o desempenho dos alunos em termos de nível de aprendizado é um bom reflexo do nível de ensino da escola (GOMES; BORGES, 2009b; AZEVEDO, 2009).

Dentre os principais achados deste estudo, destaca-se que não foram encontradas evidências estatísticas que permitam rejeitar a hipótese de que há uma relação entre a presença de tecnologia da informação nas escolas com o seu desempenho. Os 2 modelos matemáticos que foram regredidos com diferentes variáveis relacionadas à presença de tecnologia nas escolas, apresentaram boas medidas de relação com o desempenho, sendo a maior medida de relação em torno de $41 \%$. Ou seja, o modelo foi capaz de explicar $41 \%$ dos fatores que influenciam no desempenho da escola, quando este for medido pelas notas do ENEM.

Destaca-se que nos modelos a variável relacionadas ao número de computadores disponíveis para os alunos se mostrou um estimador eficiente possibilitando inclusive concluir sobre aspectos econômicos. A variável que indicou se a escola é pública ou não, se mostrou de suma importância, pois possibilitou a melhora do poder explicativo não só do modelo em que foi incluída, mas também de outras variáveis correlacionadas. Além disso, esta variável também permite inferir do ponto de vista econômico permitindo tirar conclusões importantes em relação às escolas públicas e privadas. Por exemplo, ao analisar o modelo 2, o coeficiente da variável escola pública traz evidências de que em média as escolas públicas tem 141,81 pontos a menos que as escolas privadas com os mesmos níveis de computadores, disponibilidade de internet e laboratório de informática.

Dentre as limitações presentes neste estudo, podemos destacar que em função do ENEM ter sido utilizado como medida do desempenho, este estudo limitou-se a avaliar apenas as escolas que ofertam o ensino médio, mas não se limitando a este, ou seja, escolas que ofertam o ensino fundamental e médio entraram na base, mas escolas que ofertam apenas o ensino fundamental não entraram. No entanto, destaca-se o fato de que mesmo após a retirada das escolas, foram analisadas 551 escolas do Espírito Santo, totalizando 1.417 registros de informações das escolas e do ENEM para os 3 períodos adotados no estudo.

Outra limitação deste estudo está na base do Censo, pois para os anos que foram avaliados, 2009, 2010 e 2011, só é possível obter a informação da existência ou não do recurso, mas não é possível sabermos se o recurso é utilizado, tampouco se é feito um 
bom uso. Por exemplo, consegue-se obter a informação no Censo de que a escola possui um laboratório de informática e que tem disponibilidade de internet, mas não conseguese saber se os professores realmente utilizam estes recursos com os alunos e se esse uso é ou não de qualidade, incluindo planejamento, softwares educativos, entre outras informações. Mesmo diante destas limitações, este estudo se mostra uma importante ferramenta de avaliação não só da importância do uso de tecnologia da informação em escolas, mas também de aspectos relacionados as características das escolas presentes no estudo.

Ademais, as bases de dados utilizadas, Censo e ENEM, trouxeram desafios à realização desta pesquisa, pois ambas são disponibilizadas em arquivo texto sem uso de separador entre os campos presentes nos dados. Além disso, em ambos os casos foram encontrados erros no layout dos arquivos que foram disponibilizados juntamente com as bases de dados. Felizmente, os erros nos layouts não foram impeditivos à leitura e entendimento do formato dos arquivos e os separadores foram aplicados com a ajuda de um software apropriado para esta tarefa.

A base de dados do ENEM trouxe ainda uma dificuldade adicional, pois esta base, em média, possui, em alguns casos, mais de 6 milhões de registros em um ano, sendo assim, os arquivos da base são muito grandes e os programas utilizados para a leitura dos dados não suportaram o tamanho dos arquivos. Utilizou-se então um programa de computador para separar os arquivos de texto da base do ENEM em arquivos menores para que então os dados pudessem ser lidos.

Após a leitura dos dados foi utilizado um banco de dados para cruzar as informações das escolas com as notas das escolas, este cruzamento foi possível através do campo código da escola que é o mesmo utilizado nas duas bases. Os dados foram então exportados para um software estatístico em que foram aplicados os modelos de regressão linear para a obtenção dos resultados apresentados neste estudo.

Sugere-se para estudos futuros uma análise das escolas de todo o Brasil, pois pode-se avaliar se é possível generalizar os achados para todo o país. Pode-se também aumentar o período de tempo do estudo, desde que sejam levadas em consideração questões importantes que podem ser impeditivas à inclusão de um novo período. Além disso, como discutido no estudo, as variáveis laboratório de informática e internet, não tiveram um bom desempenho como indicadores, de forma que, um novo estudo poderia realizar testes não só retirando estas variáveis do modelo, mas também incluindo novas variáveis como impressoras, projetor multimídia, entre outras.

Este estudo então ratificou, por meio de evidências estatísticas os estudos de autores como Corrêa et al. (2006), Franco et al. (2007), Valente (1997), Löbler et al. (2012) entre outros que apontaram que o uso de tecnologia da informação favorece o ensino de qualidade. Sendo assim, este estudo é uma importante ferramenta não só como base para estudos futuros, mas inclusive para tomada de decisão de agentes da gestão pública.

\section{REFERÊNCIAS}

AZEVEDO, Glauco Lívio Silva. (2009) "Eficiência do Ensino no Distrito Federal pela DEA.”, 44 f. Dissertação (Mestrado em Economia de Empresas) - Universidade Católica de Brasília, Brasília. 
BIONDI, R. L. \& FELÍCIO, F. (2007) "Atributos escolares e o desempenho dos estudantes: uma análise em painel dos dados do Saeb.”, In: Instituto Nacional de Estudos e Pesquisas Educacionais Anísio Teixeira (Inep)/MEC, Brasília, ISSN: 1414-0640.

CALDAS, Wagner K.; NOBRE, Isaura A. M.; GAVA, Tânia B. S. (2011) "Uso do Computador na Educação: Desafios Tecnológicos e Pedagógicos.", Informática na Educação: Um caminho de possibilidades e desafios. p. 15-38, Serra.

CORRÊA, A. G. D.; ASSIS, G. A.; VENÂNCIO, V.; FICHEMAN, I. K.; LOPES, R. D. (2006) "Avaliação de aceitabilidade de um computador portátil de baixo custo por criança.”, In: Simpósio Brasileiro de Informática na Educação, Brasília.

DWYER, T.; WAINE, J.; DUTRA, R. S.; COVOC, A.; MAGALHÃES, V. B.; FERREIRA, L. R.; PIMENTA, V. A.; KLEUCIO, C. (2007) "Desvendando mitos: os computadores e o desempenho no sistema escolar.", Educação e Sociedade, v. 28, n. 101, p. $1303-1328$.

FRANCO, J. F.; FICHEMAN, I. K.; AQUINO, E. M. M.; MORENO, M.; MANGERONA, M. S.; LOPES, R. D. (2007) "Usando recursos digitais como suporte para convergir e construir conhecimento.", In: Workshop de Modelos Pedagógicos em Educação a Distância: das concepções pessoais aos conceitos científicos, São Paulo.

GOMES, C. M. A.; BORGES, O. N. (2009b) “O Enem é uma avaliação educacional construtivista? Um estudo de validade de construto.”, Est. Aval. Educ, v. 20, n. 42, p. 73-88.

INEP. (2013) “Censo Escolar.”, Disponível em: http://portal.inep.gov.br/basica-censo. Acesso em: 20 nov.

LEVINE David M. et al. (2008) "Estatística: Teoria e aplicações.", 5. ed. Rio de Janeiro: Livros Técnicos e Científicos Editora.

LÖBLER, Mauri L.; LÖBLER, Laurenita M. B. (2012) "Os Laboratórios de Informática em Escolas Públicas e sua Relação com o Desempenho Escolar.”, Revista Novas Tecnologias na Educação. Porto Alegre, v. 10, n. 3, dez.

LOPES, Roseli de Deus; FICHEMAN, Irene Karaguilla; MARTINAZZO, Alexandre A. G.; CORREA, Ana G. D.; VENÂNCIO, Valkíria; BIAZON, Leandro Coletto. (2010) "O uso dos computadores e da internet em escolas públicas de capitais brasileiras.", Estudos \& Pesquisas Educacionais - Fundação Victor Civita, n. 1, p. 275, São Paulo.

NERI, M. C. (coord.). (2003) “Mapa da exclusão digital.”, Rio de Janeiro: FGV/IBRE.

TRAVITZKI, Rodrigo. (2013) "ENEM: limites e possibilidades do Exame Nacional do Ensino Médio enquanto indicador de qualidade escolar.", $320 \mathrm{f}$. Tese (Doutorado em Educação) - Faculdade de Educação, Universidade de São Paulo.

VALENTE, J. A. (1997) "O uso inteligente do computador na educação.", Pátio Revista Pedagógica. Editora: Artes Médicas Sul, ano 1, n. 1, p. 19-21.

WOOLDRIDGE, J. M. (2006) "Introdução à Econometria.", São Paulo: Thomson Learning. 